\title{
ALTERNATIVE VERSION OF SHAPLEY'S THEOREM ON CLOSED COVERINGS OF A SIMPLEX
}

\author{
TATSURO ICHIISHI
}

(Communicated by Paul S. Muhly)

\begin{abstract}
Sperner's theorem as formulated by Ky Fan is dual to the KnasterKuratowski-Mazurkiewicz theorem. Shapley's theorem is a generalization of the Knaster-Kuratowski-Mazurkiewicz theorem. This paper points out that Shapley's theorem is a generalization of Sperner's theorem as well, by establishing an alternative version of Shapley's theorem. Applications to the multiperson cooperative game theory are also discussed.
\end{abstract}

1. Introduction. Let $N$ be a finite set, and let $\left\{e^{j}\right\}_{j \in N}$ be an affinely independent subset of a vector space indexed by the members of $N$. Denote by $\mathscr{N}$ the family of nonempty subsets of $N$ (i.e., $\mathscr{N}:=2^{N} \backslash\{\varnothing\}$ ), and define $\Delta^{S}:=\operatorname{co}\left\{e^{j} \mid j \in S\right\}$ (the convex hull of $\left\{e^{j} \mid j \in S\right\}$ ), and $m^{S}:=\sum_{j \in S} e^{j} / \# S$, for each $S \in \mathscr{N}$. The simplex $\Delta^{N}$ is endowed with the unique Hausdorff linear-space topology for a finite dimensional vector space (i.e., with the Euclidean topology). The classical theorem by Knaster-Kuratowski-Mazurkiewicz [9], which is equivalent to Brouwer's fixed-point theorem [1], says:

THE K-K-M THEOREM [9]. Let $\left\{C^{j}\right\}_{j \in N}$ be a family of closed subsets of $\Delta^{N}$ such that $\Delta^{S} \subset \bigcup_{j \in S} C^{j}$ holds for each $S \in \mathscr{N}$. Then $\bigcap_{j \in N} C^{j} \neq \varnothing$.

The dual form to the K-K-M theorem may be found in Sperner [14], which was reformulated by Fan [2] as:

SPERNER'S THEOREM [14]. Let $\left\{C^{j}\right\}_{j \in N}$ be a closed covering of $\Delta^{N}$ such that $\Delta^{N \backslash\{j\}} \subset C^{j}$ holds for each $j \in N$. Then $\bigcap_{j \in N} C^{j} \neq \varnothing$.

A subfamily $\mathscr{B}$ of $\mathscr{N}$ is called balanced, if $m^{N} \in \operatorname{co}\left\{m^{S} \mid S \in \mathscr{B}\right\}$. Shapley [12] established a generalization of the K-K-M theorem:

SHAPLEY'S THEOREM [12]. Let $\left\{C^{S}\right\}_{S \in \mathcal{N}}$ be a family of closed subsets of $\Delta^{N}$ such that $\Delta^{T} \subset \bigcup_{S \subset T} C^{S}$ holds for each $T \in \mathscr{N}$. Then there exists a balanced family $\mathscr{B}$ for which $\bigcap_{S \in \mathscr{B}} C^{S} \neq \varnothing$.

Shapley [12] proved his theorem by using the "path-following technique" of Lemke-Howson [10]. For an alternative proof which makes use of Brouwer's fixedpoint theorem [1] and a sequence of simplicial partitions (makes use of Kakutani's fixed-point theorem [7], makes use of Ky Fan's coincidence theorem [3], resp.), see Todd [15 and 16] (Shapley [13], Ichiishi [5], resp.). The first purpose of this paper is to point out that Shapley's theorem can be reformulated as follows.

Received by the editors August 20, 1987 and, in revised form, October 26, 1987.

1980 Mathematics Subject Classification (1985 Revision). Primary 47H10; Secondary 90D12. 
Alternative Version OF SHAPLEY'S THEOREM. Let $\left\{C^{S}\right\}_{S \in \mathscr{N}}$ be a closed covering of $\Delta^{N}$ such that $\Delta^{T} \subset \bigcup_{S \supset N \backslash T} C^{S}$ holds for each $T \in \mathscr{N}$. Then there exists a balanced family $\mathscr{B}$ for which $\bigcap_{S \in \mathscr{B}} C^{S} \neq \varnothing$.

It is easy to check that the alternative version generalizes Sperner's theorem.

Shapley [12] applied his theorem to give another proof of Scarf's [11] theorem for nonemptiness of the core; the latter theorem is now considered one of the fundamental theorems in the multiperson cooperative game theory. Recently, Keiding and Thorlund-Petersen [8] and Vohra [17] provided new alternative proofs of the Scarf theorem by using the K-K-M theorem and Kakutani's fixed-point theorem, respectively. The second purpose of the present paper is to point out that both Keiding and Thorlund-Petersen [8] and Vohra [17] were looking at closed coverings of the type given in the alternative version of Shapley's theorem, and that the Scarf theorem follows directly from the alternative version.

2. Proofs of the alternative version of Shapley's theorem. Two proofs will be presented here. The first proof (due to David Schmeidler) shows that Shapley's theorem implies the alternative version, and conversely the alternative version implies Shapley's theorem. Thus, given Shapley's theorem, the alternative version is established. The second proof shows that the alternative version is a simple consequence of Ky Fan's coincidence theorem.

Equivalence of Shapley's theorem and the alternative version (Schmeidler). Observe first that if $\mathscr{B}(\subset \mathscr{N})$ is a balanced family such that $N \notin \mathscr{B}$, then the family $\{N \backslash S \mid S \in \mathscr{B}\}$ is a balanced family. Indeed, set $\chi_{S}:=\sum_{j \in S} e^{j}$. The family $\mathscr{B}$ is balanced iff there exists $\left(\lambda_{S}\right)_{S \in \mathscr{B}} \subset \mathbf{R}_{+}$such that $\sum_{S \in \mathscr{B}} \lambda_{S} \chi_{S}=\chi_{N}$. Since $N \notin \mathscr{B}, \lambda:=\sum_{S \in \mathscr{B}} \lambda_{S}>1$. Notice

$$
\begin{aligned}
\sum_{S \in \mathscr{B}} \lambda_{S} \chi_{N \backslash S} & =\sum_{S \in \mathscr{B}} \lambda_{S}\left(\chi_{N}-\chi_{S}\right) \\
& =\lambda \chi_{N}-\sum_{S \in \mathscr{B}} \lambda_{S} \chi_{S}=(\lambda-1) \chi_{N}
\end{aligned}
$$

so

$$
\sum_{S \in \mathscr{B}} \frac{\lambda_{S}}{\lambda-1} \chi_{N \backslash S}=\chi_{N} .
$$

Consequently, the family $\{N \backslash S \mid S \in \mathscr{B}\}$ is balanced.

(1) Now, given Shapley's theorem, let $\left\{C^{S}\right\}_{S \in \mathcal{N}}$ be a closed covering of $\Delta^{N}$ such that $\Delta^{T} \subset \bigcup_{S \supset N \backslash T} C^{S}$ holds for each $T \in \mathscr{N}$. If $C^{N} \neq \varnothing$, there is nothing to prove; so assume $C^{N}=\varnothing$. Define $D^{S}:=C^{N \backslash S}$ for every $S \in \mathscr{N} \backslash\{N\}$, and $D^{N}:=\varnothing$. Then, $\Delta^{T} \subset \bigcup\left\{D^{S} \mid \varnothing \neq S \subset T\right\}$ for each $T \in \mathscr{N}$. By Shapley's theorem, there exists a balanced family $\mathscr{B}$ such that $\bigcap_{S \in \mathscr{B}} D^{S} \neq \varnothing$. Since $N \notin \mathscr{B}$, the family $\mathscr{C}:=\{N \backslash S \mid S \in \mathscr{B}\}$ is balanced by the observation at the outset of this proof. Notice

$$
\bigcap_{S \in \mathscr{C}} C^{S}=\bigcap_{N \backslash S \in \mathscr{B}} D^{N \backslash S} \neq \varnothing .
$$

(2) One can prove the converse implication analogously. Q.E.D.

Proof of the alternative version by Ky Fan's coincidence theorem. Without loss of generality, one may assume that $\Delta^{N}$ is a subset of $\mathbf{R}^{N}$. The relative interior 
of a subset $A$ of $\mathbf{R}^{N}$ (i.e., the interior of $A$ relative to its affine hull) is denoted by ri $A$. Define for each $x \in \Delta^{N}, I(x):=\left\{S \in \mathscr{N} \mid C^{S} \ni x\right\}, F(x):=\left\{m^{N}\right\}$, and $G(x):=\operatorname{co}\left\{m^{S} \mid S \in I(x)\right\}$. The correspondences (set-valued maps) $F$ and $G$ are upper semicontinuous in $\Delta^{N}$, and are nonempty-, compact- and convex-valued.

Choose any $T \in \mathscr{N}$, any $x \in$ ri $\Delta^{T}$, and any $p \in \mathbf{R}^{N}$ such that the inner product $p \cdot x=\min p \cdot \Delta^{N}$. Under the assumption of the alternative version, there exists $S \in \mathscr{N}$ such that $S \supset N \backslash T$ and $x \in C^{S}$. If $S=N$, there is nothing to prove; so assume $S \varsubsetneqq N$. On one hand, the choice of $x$ and $p$ implies $p \cdot y=\min p \cdot \Delta^{N}$ for every $y \in \Delta^{T}$. In particular, (since $\varnothing \neq N \backslash S \subset T$ )

$$
p \cdot m^{N \backslash S}=\min p \cdot \Delta^{N} .
$$

On the other hand, (since $\varnothing \neq S \varsubsetneqq N$ ) there exists $\alpha, 0<\alpha<1$, such that

$$
p \cdot m^{N}=\alpha p \cdot m^{S}+(1-\alpha) p \cdot m^{N \backslash S} \text {. }
$$

By (1) and (2), $p \cdot m^{N} \leq p \cdot m^{S}$; that is, there exist $u \in F(x)$ and $v \in G(x)$ such that $p \cdot u \leq p \cdot v$.

Thus, all the assumptions of Ky Fan's coincidence theorem [4, Theorem 5, p. 108] are satisfied. So, there exists $x^{*} \in \Delta^{N}$ for which $F\left(x^{*}\right) \cap G\left(x^{*}\right) \neq \varnothing$. Family $I\left(x^{*}\right)$ is the required balanced family. Q.E.D.

3. Applications to game theory. The finite set $N$ is now interpreted as the set of players, and $\mathcal{N}$ as the family of nonempty coalitions. A non-side-payment game is a correspondence (set-valued map) $V: \mathscr{N} \rightarrow \mathbf{R}^{N}$ such that $\left[u, v \in \mathbf{R}^{N}\right.$, $\left.\forall j \in S: u_{j}=v_{j}\right]$ implies $[u \in V(S)$ iff $v \in V(S)]$ for every $S \in \mathcal{N}$. Set $V(S)$, or rather its projection to $\mathbf{R}^{S}$, is interpreted as the set of utility allocations attainable in coalition $S$. The core of a non-side-payment game $V$ is a typical solution concept, and is defined as the set $C(V)$ of all $u \in \mathbf{R}^{N}$ such that (a) $u \in V(N)$ and (b) it is not true that there exist $S \in \mathscr{N}$ and $u^{\prime} \in V(S)$ such that $u_{j}<u_{j}^{\prime}$ for every $j \in S$. Condition (a) is the feasibility of the utility allocation via the grand coalition $N$, and condition (b) is the coalitional stability of $u$. A non-side-payment game $V$ is called balanced if for every balanced subfamily $\mathscr{B}$ of $\mathscr{N}$, it follows that $\bigcap_{S \in \mathscr{B}} V(S) \subset V(N)$. See, e.g., Ichiishi [6, Chapter 5] for further discussions. The following version of Scarf's theorem is weaker than the original version [11], but the latter follows straightforwardly from the former. The interior (closure, boundary, resp.) of a set $A$ in $\mathbf{R}^{N}$ is denoted by $\AA(\bar{A}, \partial A$, resp.).

SCARF'S THEOREM [11]. Let $V: \mathscr{N} \rightarrow \mathbf{R}^{N}$ be a non-side-payment game. Assume: (i) $V(S)-\mathbf{R}_{+}^{N}=V(S)$, for every $S \in \mathscr{N}$; (ii) There exists $M \in \mathbf{R}$ such that for every $S \in \mathscr{N}$, every $u \in V(S) \cap \mathbf{R}_{+}^{N}$ and every $j \in S$, it follows that $u_{j}<M$; (iii) $0 \in \stackrel{\circ}{V}(S)$ for every $S \in \mathscr{N}$; (iv) $V(S)$ is closed in $\mathbf{R}^{N}$ for every $S \in \mathscr{N}$; and (v) $V$ is balanced. Then $C(V)$ is nonempty.

Remark that when proving Scarf's theorem, Shapley [12] applied his theorem to a suitable simplex contained in the negative cone, $-\mathbf{R}_{+}^{N}$. The proofs in the present section, on the other hand, apply the alternative version of Shapley's theorem to a simplex contained in the positive cone, $\mathbf{R}_{+}^{N}$. Indeed, throughout this section the vector $e^{j} \in \mathbf{R}^{N}$ is given by: $e_{i}^{j}:=0$ if $i \neq j$, and $e_{j}^{j}:=1$. The set $\Delta^{N}$ is then the unit simplex. 
The Keiding and Thorlund-Petersen approach. For each $\varepsilon>0$, consider the new game $V_{\varepsilon}$ defined by: $V_{\varepsilon}(S):=V(S)$ if $S \neq N$, and $V_{\varepsilon}(N):=\left\{u \in \mathbf{R}^{N} \mid \rho(u, V(N)) \leq\right.$ $\varepsilon\}$, where $\rho(u, A):=\inf \{\|u-a\| \mid a \in A\}$. Define for each $S \in \mathscr{N}$,

$$
G_{\varepsilon}^{S}:=\left\{\begin{array}{l|l}
x \in \Delta^{N} & \begin{array}{l}
\left(\forall u \in V_{\varepsilon}(N): u / \sum_{j \in N} u_{j}=x\right): \\
\exists v \in V_{\varepsilon}(S): \forall j \in N: v_{j}>u_{j}
\end{array}
\end{array}\right\} .
$$

Suppose $C(V)=\varnothing$. Then, by compactness of $V(N) \cap \mathbf{R}_{+}^{N}$, one may choose $\varepsilon>0$ for which $C\left(V_{\varepsilon}\right)=\varnothing$. Keiding and Thorlund-Petersen [8] looked at the family $\left\{G_{\varepsilon}^{S}\right\}_{S \in \mathcal{N}}$, given this $\varepsilon$. Now, it is easy to check

$$
\Delta^{N} \subset \bigcup_{S \in \mathscr{N} \backslash\{N\}} G_{\varepsilon}^{S}, \quad \text { and } \quad \forall T \in \mathscr{N} \backslash\{N\}: \Delta^{T} \subset G_{\varepsilon}^{N \backslash T} .
$$

Condition (3) is valid even when $\overline{G_{\varepsilon}^{S}}$ replaces $G_{\varepsilon}^{S}$ for all $S$. In particular, all the assumptions in the alternative version hold true for family $\left\{\overline{G_{\varepsilon}^{S}}\right\}_{S \in \mathscr{N}}$ of subsets of $\Delta^{N}$ (here, $\left.\overline{G_{\varepsilon}^{N}}:=\varnothing\right)$. So,

$$
\text { ( } \exists \mathscr{B}: \text { balanced) }: \exists x^{*} \in \bigcap_{S \in \mathscr{B}} \overline{G_{\varepsilon}^{S}} .
$$

Let $u^{*} \in \partial V_{\varepsilon}(N)$ be the unique vector such that $x^{*}=u^{*} / \sum_{j \in N} u_{j}^{*}$. By closedness of $V(S), u^{*} \in \bigcap_{S \in \mathscr{B}} V(S)$. Since $V$ is balanced, $u^{*} \in V(N)$, which contradicts the fact that $u^{*} \in \partial V_{\varepsilon}(N)$. Q.E.D.

The Vohra approach. Define $W:=\left\{u \in \bigcup_{S \in \mathcal{N}} V(S) \mid \forall j \in N:-M \leq u_{j} \leq M\right\}$, and for each $x \in \Delta^{N}$, consider the unique point $f(x) \in \partial W$ defined by: $\exists t \geq$ $0: f(x)=t x$. The function $f: \Delta^{N} \rightarrow \partial W$ is continuous. Define $C^{S}:=\{x \in$ $\left.\Delta^{N} \mid f(x) \in V(S)\right\}$. Vohra [17] looked at the family $\left\{C^{S}\right\}_{S \in \mathscr{N}}$. Now, it is easy to check that $\left\{C^{S}\right\}_{S \in \mathcal{N}}$ is a closed covering of $\Delta^{N}$ such that $\Delta^{T} \subset C^{N \backslash T}$ for every $T \in \mathscr{N} \backslash\{N\}$. In particular, all the assumptions in the alternative version hold true. So, ( $\exists \mathscr{B}$ : balanced) : $\exists x^{*} \in \bigcap_{S \in \mathscr{B}} C^{S}$. In view of the balancedness assumption on $V$, one concludes $f\left(x^{*}\right) \in C(V)$. Q.E.D.

ACKNOWLEDGMENT. This is a revised version of: Tatsuro Ichiishi, Dual result to Shapley's theorem on closed coverings of a simplex, Working Paper No. 87-04, Department of Economics, Ohio State University, August 1987. My interest in the research reported in this paper arose one evening when I had very stimulating discussions with Professors Ky Fan, Lloyd S. Shapley, and Rajiv Vohra on the fixed-point literature and the core-nonemptiness problems during the International Conference on Game Theory and Applications, Ohio State University, June 18-24, 1987. I would like to express my deep gratitude to Professors Fan, Shapley and Vohra. Credit for the insight that led to this paper goes to Professor Ky Fan, whom I would also like to thank for his encouragement, guidance and comments. After writing up the first version (August 1987), David Schmeidler pointed out the equivalence of Shapley's theorem and (the theorem that is called in the present version as) the Alternative Version of Shapley's theorem. I would like to thank Professor Schmeidler for allowing me to reproduce his argument here.

\section{REFERENCES}

1. L. E. J. Brouwer, Über Abbildungen von Mannifaltigkeiten, Math. Ann. 71 (1912), 97-115.

2. K. Fan, A covering property of simplexes, Math. Scand. 22 (1968), 17-20. 
3. __ Extensions of two fixed point theorems of F. E. Browder, Math. Z. 112 (1969), 234-240.

4. __ A minimax inequality and applications (O. Shisha, ed.), Inequalities. III, Academic Press, New York, 1972, pp. 103-113.

5. T. Ichiishi, On the Knaster-Kuratowski-Mazurkiewicz-Shapley theorem, J. Math. Anal. Appl. 81 (1981), 297-299.

6. __ Game theory for economic analysis, Academic Press, New York, 1983.

7. S. Kakutani, A generalization of Brouwer's fixed-point theorem, Duke Math. J. 8 (1941), 457459.

8. H. Keiding and L. Thorlund-Petersen, The core of a cooperative game without side payments, mimeo, August 1985.

9. B. Knaster, C. Kuratowski, and S. Mazurkiewicz, Ein Beweis des Fixpunktsatzes für n-dimensionale Simplexe, Fund. Math. 14 (1929), 132-137.

10. C. E. Lemke and J. T. Howson, Equilibrium points of bi-matrix games, SIAM J. Appl. Math. 12 (1964), 413-423.

11. H. Scarf, The core of an n-person game, Econometrica 35 (1967), 50-69.

12. L. S. Shapley, On balanced games without side payments, Mathematical Programming (T. C. Hu and S. M. Robinson, Eds.), Academic Press, New York, 1973, pp. 261-290.

13. __ Lecture Notes, Dept. of Math., Univ. of California, Los Angeles, 1987.

14. E. Sperner, Neuer Beweis für die Invarianz der Dimensionszahl und des Gebietes, Abh. Math. Sem. Univ. Hamburg 6 (1928), 265-272.

15. M. Todd, Lecture Notes, School of Operations Research and Industrial Engineering, Cornell Univ., Ithaca, 1978.

16. __ Private communication, 1979.

17. R. Vohra, On Scarf's theorem on the non-emptiness of the core: A direct proof through Kakutani's fixed-point theorem, Working Paper No. 87-2, Dept. of Economics, Brown Univ., January 1987. Revised: June 1987.

Department of Economics, Ohio State University, ARPS hall, 1945 North High STREeT, Columbus, OHIO 43210-1172 